\title{
Editorial
}

\section{Green Intelligent Transport System}

\author{
Wuhong Wang, ${ }^{1}$ Geert Wets, ${ }^{2}$ Heiner Bubb, ${ }^{3}$ and Huimin Niu ${ }^{4}$ \\ ${ }^{1}$ Department of Transportation Engineering, Beijing Institute of Technology, Beijing 100081, China \\ ${ }^{2}$ Transportation Research Institute (IMOB), Hasselt University, 3590 Diepenbeek, Belgium \\ ${ }^{3}$ Lehrstuhl für Ergonomie, Technische Universität München, 85747 Munich, Germany \\ ${ }^{4}$ School of Traffic and Transportation, Lanzhou Jiaotong University, Lanzhou 730070, China
}

Correspondence should be addressed to Wuhong Wang; wangwuhong@bit.edu.cn

Received 29 April 2014; Accepted 29 April 2014; Published 14 May 2014

Copyright (C) 2014 Wuhong Wang et al. This is an open access article distributed under the Creative Commons Attribution License, which permits unrestricted use, distribution, and reproduction in any medium, provided the original work is properly cited.

The transportation system is a complex dynamic system with various elements such as drivers, passengers, pedestrians, vehicles, and roads. Recently, with the rapid development of motorization, transportation problems (i.e., accidents, congestions, and pollutions) have attracted much attention from the public. Not only are these problems highly relevant to our daily life, but also they provide interesting phenomena and talking-points. For example, traffic congestion arouses great concern and it shows complex dynamical characteristics. The phenomenon of phase transitions has been found in the congested flow from the perspective of statistical mechanics and nonlinear dynamics. Moreover, the scientists and engineers are seeking solutions for improving the performance and safety of transportation system operation by the way of interdisciplinary studies. In this context, some great efforts have been made in the field of intelligent transportation systems (ITS), which has been regarded as the most effective method and advanced technology for reaching a more efficient and safe road usage.

The term ITS is used to describe a set of concepts and technologies for a kind of transportation systems while the main focus is on the integration of information and communication technologies (ICT) with infrastructure, vehicles, and traffic users. ITS aims to provide some innovative services for establishing harmonious and smart transportation system in which various traffic users can travel more safer and faster. A typical feature of ITS is the versatile nature of new technique. At present, wireless communications, computational technologies, sensing technologies, and pattern recognition have played a crucial role in the development of
ITS. Additionally, predictive techniques have been developed to provide advanced service for participants in transportation system. Of the various subsystems of ITS, for instance, advanced driver assistance system (ADAS) can improve efficiency and safety of vehicle operation by compensating for the unfavorable human behavior.

Nowadays, the concentration on low carbon, energy saving, and pollution control is increasing in the field of ITS. There are a number of beneficial forms of such green transportation that make our lives easier and more fun. Green transportation involves effective and efficient resource utilization, changes in transportation structure, and improving transportation environment. Green ITS will be the objective of development of transportation system in the future. Green ITS can make a positive contribution to the social, economic, and environmental sustainability in urban system. It can also provide harmonious connections between human and vehicles as well as infrastructures.

The focus of this special issue is to present the new achievements in basic and applied research relating to ITS and reveal the complex regularity of transportation system. The aim of this special issue is to contribute impotently to the development of green ITS, especially for the strategies for improving traffic performance and safety. This special issue invites original research from the theoretical and practical point of view. The topics of this special issue include the following:

intelligent transportation system,

discrete and dynamic problems in transportation system, 
application of chaos in traffic flow,

driving behavior and driver assistance system,

discrete optimization methods in ITS,

transit and rail systems operation,

environment protection and energy saving in transportation,

transportation policy and economics.

Wuhong Wang

Geert Wets

Heiner Bubb

Huimin Niu 


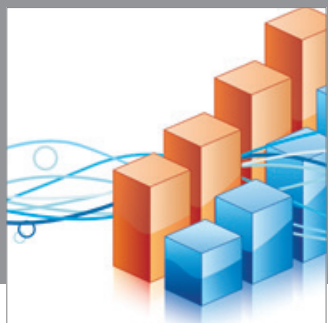

Advances in

Operations Research

mansans

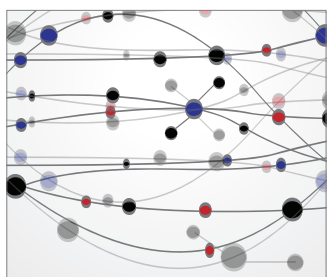

The Scientific World Journal
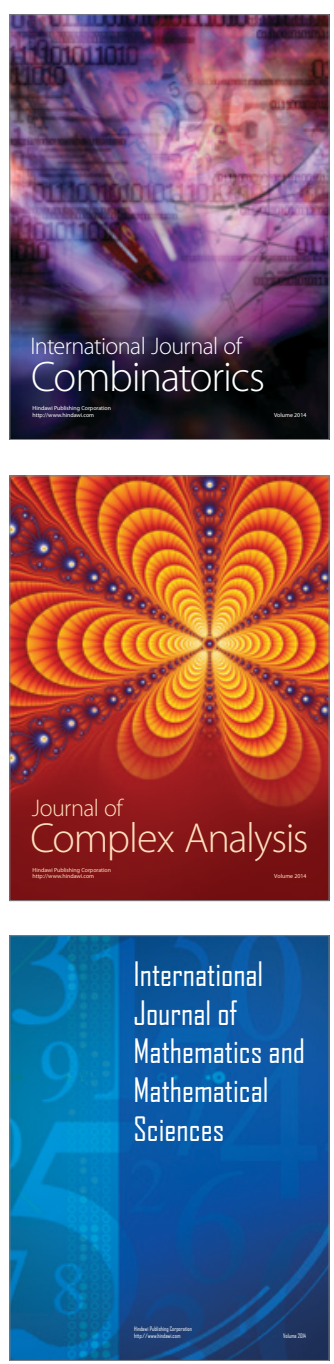
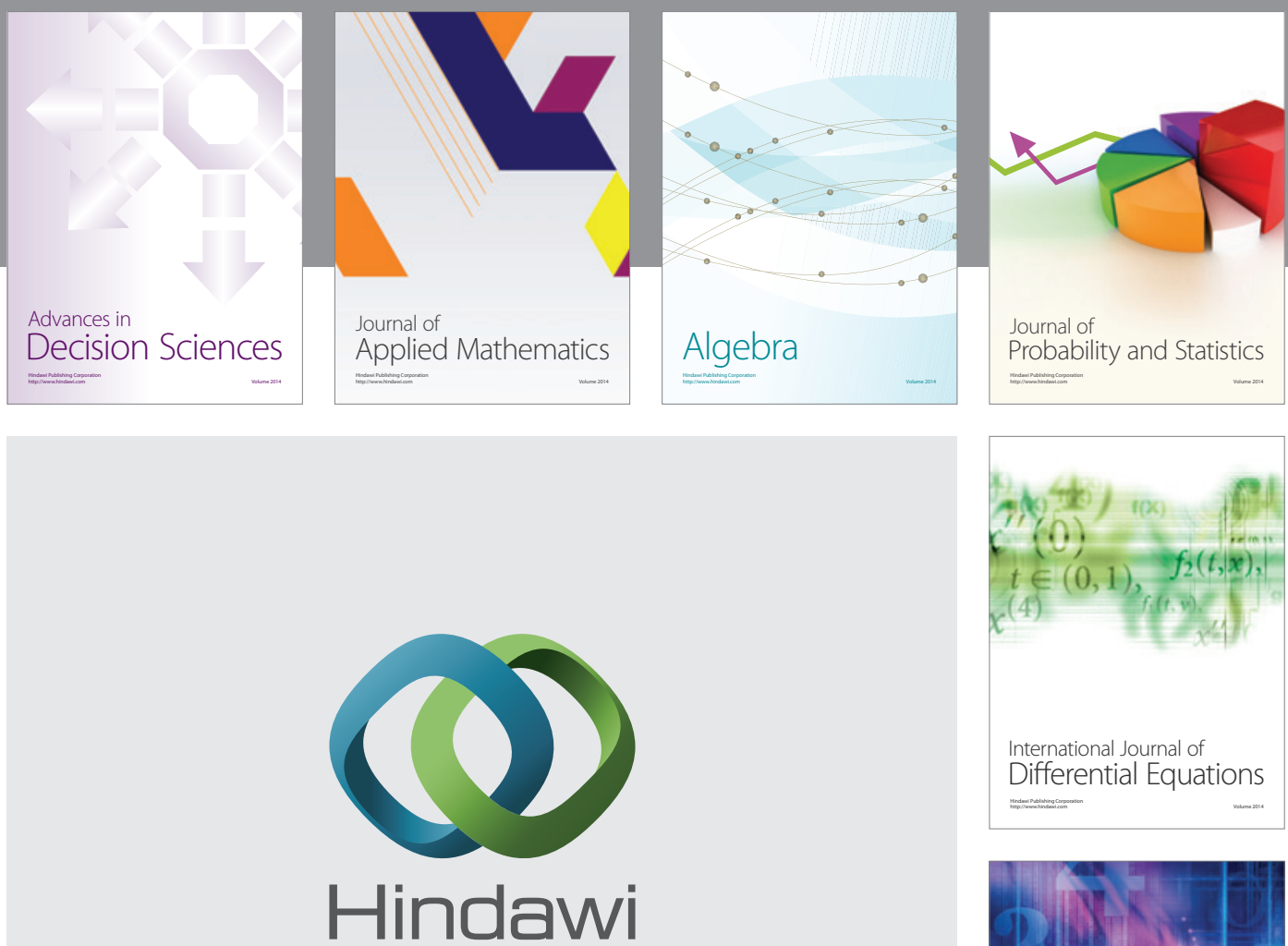

Submit your manuscripts at http://www.hindawi.com
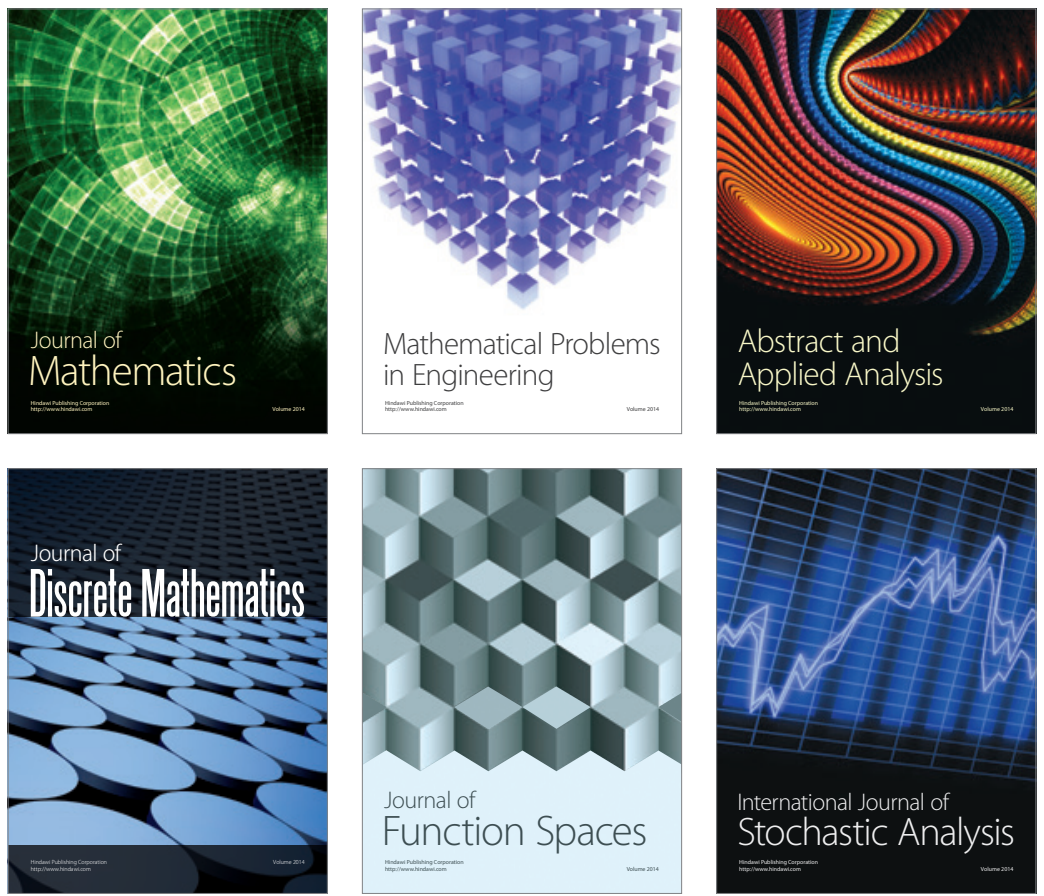

Journal of

Function Spaces

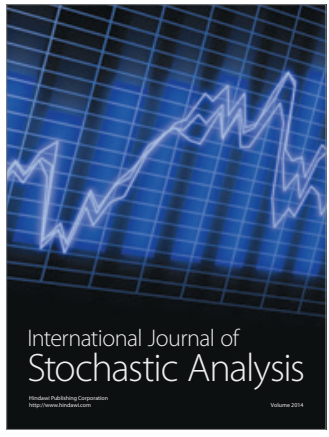

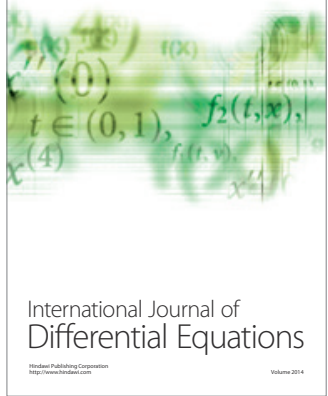
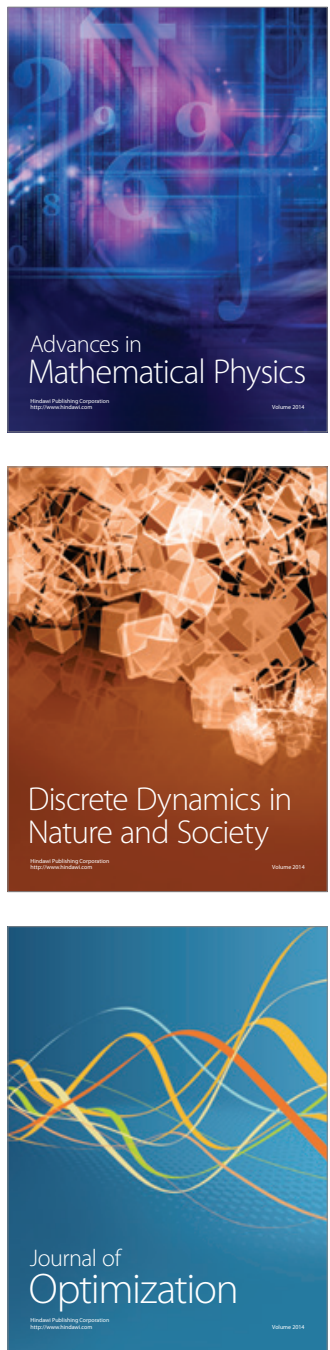